\title{
Research on Reflection and Transmission at Boundaries in Ultrasonic Testing
}

\author{
L. Zhang ${ }^{1, a}$, G. H. Wang ${ }^{1}$, J. J. Lu ${ }^{2}$ \\ ${ }^{1}$ School of Physics, Tonghua Normal University, Tonghua 134002, China \\ ${ }^{2}$ School of Material Sciences and Engineering, Hebei University of Technology, Tianjin, China \\ azhangli4546@yeah.net
}

Keywords: reflection, transmission, ultrasonic testing.

\begin{abstract}
Surface(Rayleigh)wave are induced at solid-gas or solid-liquid interfaces, when the probe is at the second critical angle setting; The surface wave profile is mainly transverse and so the surface wave is approximately a shear wave at its critical angle. The variation of sound velocities with frequency is very small (non dispersive) over the frequency range used in NDT.
\end{abstract}

\section{Introduction}

Since these original inventions, ultrasonic technology has advanced greatly. Modern technologies include immersion systems as well as many imaging techniques, including tomography reconstructions and acoustic microscopes. Another important development is the tone-burst system, which allows the operator to take greater control of testing by specifying the signal to be applied to the transducer.

\section{Formulation}

In nondestructive testing ${ }^{[1-3]}$, boundaries ${ }^{[4-5]}$ where there are discontinuities in $\mathrm{Z}$ are detected because they cause the ultrasonic waves to be reflected. The fraction of the incident intensity in the reflected waves can be derived because the particle velocity $\mathrm{Q}$ and local particle pressure $\mathrm{P}$ are required to be continuous across the boundary. That is;

$$
\mathrm{Q}_{i}+\mathrm{Q}_{r}=\mathrm{Q}_{t}
$$

Where $\mathrm{Q}_{i}, \mathrm{Q}_{r}$ and $\mathrm{Q}_{t}$ are the particle velocities at the boundary of the incident, reflected, and transmitted waves. Again;

$$
\mathrm{P}_{i}+\mathrm{P}_{r}=\mathrm{P}_{t}
$$

Where $\mathrm{P}_{i}, \mathrm{P}_{r}$ and $\mathrm{P}$ are the local acoustic pressure at boundary associated with the incident, reflected, and transmitted waves.

This leads to the derivation, by Poisson in the early $19^{\text {th }}$ century, of the fraction, of the energy reflected $\mathrm{R}_{E}$ normal to the boundary between regions of specific acoustic impedance $\mathrm{Z}_{1}$ and $\mathrm{Z}_{2}$ to be;

$$
\mathrm{R}_{B}=\left[\frac{Z_{2}-Z_{1}}{Z_{2}+Z_{1}}\right]^{2}
$$

And the fraction of the energy transmitted $\mathrm{T}_{E}$ at the boundary.

$$
\mathrm{T}_{B}=1-\mathrm{R}_{B}=\frac{4 Z_{1} Z_{2}}{\left(Z_{1}+Z_{2}\right)^{2}}
$$

The boundary length is taken to be much greater than the sound wavelength. For waves incident obliquely at an angle $\alpha_{1}$ (less than the first critical angle), it was shown by Green in 1838 that the fraction of the energy reflected $\mathrm{R}_{E}{ }^{\prime}$ is given by

$$
\mathrm{R}_{E}^{\prime}=\left[\frac{Z_{2} \cos \alpha_{1}-Z_{1} \cos \alpha_{2}}{Z_{2} \cos \alpha_{1}+Z_{1} \cos \alpha_{2}}\right]^{2}
$$


( $\mathrm{R}_{E}{ }^{\prime}$ is a real number if $\alpha_{1}<$ the first critical angle; $\mathrm{R}_{E}{ }^{\prime}$ is a complex number if the first critical angle low. <second critical angle; $\mathrm{R}_{B}{ }^{\prime}$ is an imaginary number if $\alpha_{1}>$ the second critical angle) and the fraction transmitted $\mathrm{T}_{B}{ }^{\prime}$ is given by

$$
\mathrm{T}_{B}^{\prime}=\frac{4 Z_{2} Z_{1} \cos \alpha_{1} \cos \alpha_{2}}{\left(Z_{2} \cos \alpha_{1}+Z_{1} \cos \alpha_{2}\right)^{2}}
$$

Similar to $\mathrm{R}_{E}$ ', $\mathrm{T}_{B}$ ' is a real, complex, or imaginary number depending on whether $\alpha_{1}$ is less than the first critical angle, between the first and second critical angle, or greater than the second critical angle.

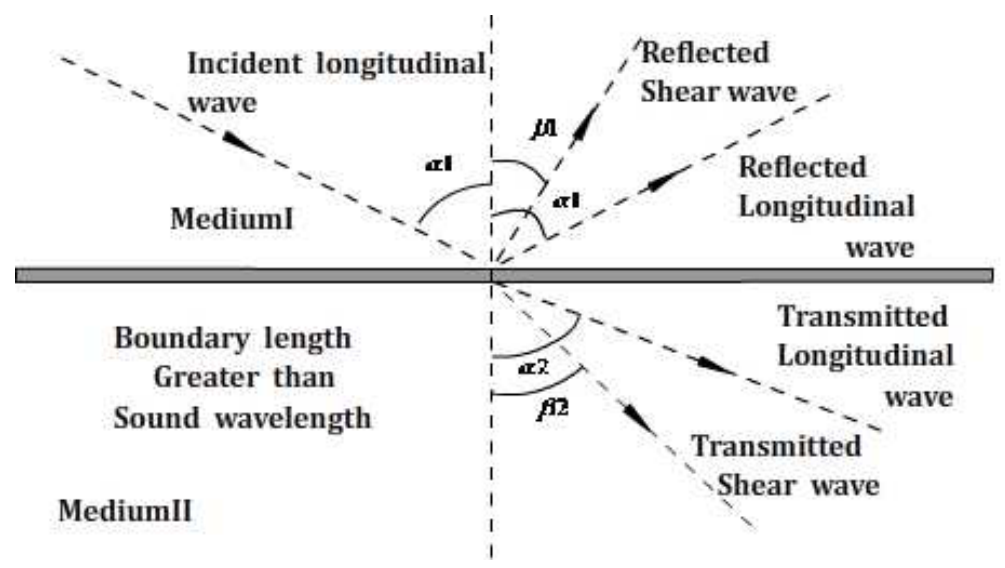

Figure 1 Simplified case of no-wave-mode conversion

Figure 1 shows a simplified case of the reflection of an acoustic wave incident on a planar boundary between two solid media of different specific acoustic impedances $Z_{1}$ and $Z_{2}$. The incident and reflected waves are inclined at the same angle $\alpha_{1}$, different from $\alpha_{2}$, the angle of the transmitted beam, as shown. The angles $\alpha_{1}$ and $\alpha_{2}$ are given by Snell's Law as

$$
\frac{\sin \alpha_{1}}{\sin \alpha_{2}}=\frac{V_{1}}{V_{2}}
$$

Where $\mathrm{V}_{1}$ and $\mathrm{V}_{2}$ are the wave velocities in medium I and II, respectively. The wave velocity of incident and reflected waves $V_{1}$ are the same in medium $\mathrm{I}$, but different from the velocity $\mathrm{V}_{2}$ in medium II.It is assumed that the sound wavelength $\lambda$ is much smaller than the size of the boundary. For density $\rho_{1}$ in medium I, and $\rho_{2}$ in medium II , $\mathrm{Z}_{1}=\rho_{1} \mathrm{~V}_{1}$ and $\mathrm{Z}_{2}=\rho_{2} \mathrm{~V}_{2}$ (Wave conversion is described in Figure 2).

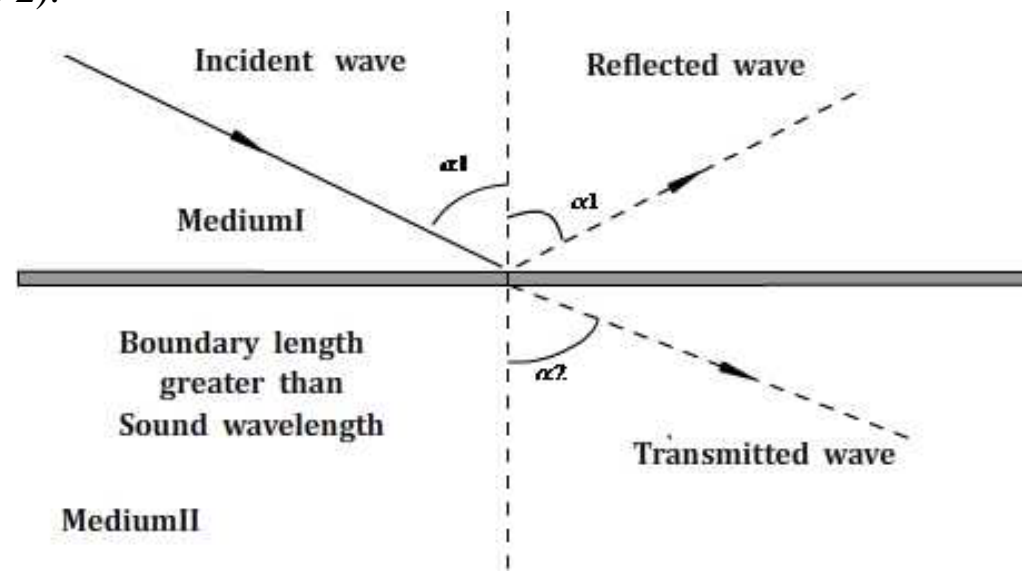

Figure 2 Wave mode conversion at a boundary

\section{Wave Mode Conversion at Boundaries}


When a longitudinal wave arrives at the boundary, as shown in Figure 2, a longitudinal wave is reflected, and also there is a component of the longitudinal wave that is transmitted across the boundary, traveling with velocity $\mathrm{V}_{L 2}$ in the second region. The angles to the normal $\alpha_{1} \alpha_{2}$ as shown in Figure 1 and Figure 2 are given by Snell's Law to be

$$
\frac{\sin \alpha_{2}}{\sin \alpha_{1}}=\frac{V_{L 2}}{V_{L 1}}
$$

What also happens at the boundary is that a component of the longitudinal sound wave is converted into a shear wave, with a shear wave being reflected in region 1 and a shear wave refracted in region 2...A shear wave is generated at the boundary in reflection and in transmission as shown in Figure 2, with different shear velocities in the two regions $\mathrm{V}_{S 1}$ and $\mathrm{V}_{s 2}$. The angles $\beta_{1}$ and $\beta_{2}$ are given by Snell's Law to be

$$
\frac{\sin \beta_{1}}{\sin \beta_{2}}=\frac{V_{S 1}}{V_{S 2}}
$$

Longitudinal wave velocities are greater than shear wave velocities, so that their relative angular positions are as shown. Figure 2 should be compared to Figure 1 in which mode conversion was excluded for the sake of simplicity.

There is an angle of critical incidence where the longitudinal refraction angle $\alpha_{2}$ is $90^{\circ}$, so that the longitudinal wave does not penetrate into region 2 across the boundary.

This condition is given by

$$
\frac{\sin \alpha_{1}}{\sin 90^{\circ}}=\sin \alpha_{1}=\frac{V_{L 1}}{V_{L 2}}
$$

When the longitudinal wave is totally internally so that only the shear wave will penetrate the transducer generating longitudinal waves is set at an angle greater than the critical angle, and so only the shear wave will penetrate into the specimen. As shown in Figure 2, Plexiglas's is often used on a steel specimen; the critical angle of total reflection of the longitudinal waves is $27.5^{\circ}$.This is referred to as the first critical angle.

There is a second critical angle when the shear wave is also totally internally reflected and no sound wave penetrates into the specimen. This occurs when

$$
\frac{\sin \alpha_{1}}{\sin 90^{\circ}}=\sin \alpha_{1}=\frac{V_{L 1}}{V_{S 2}}
$$

Critical incident angles are for total internal reflection for longitudinal and for shear waves in several materials using a Plexiglas's wedge and also for immersion testing. The first critical angle is for longitudinal waves, and the second critical angle is for transverse waves. When a pure transverse wave is required in the test piece, the angle of incidence used should be between the first and second critical angles. To obtain only a transverse wave in steel using a plexiglass wedge, the incident angle in the transducer should be about $40^{\circ}$.

The critical angles for steel are:

First critical angle for steel by water immersion test

$\operatorname{Arcsin}\left[\frac{V_{\mathrm{LH}_{2} \mathrm{O}}}{V_{\text {Lsteel }}}\right]=\arcsin \frac{1.49 \mathrm{~km} / \mathrm{s}}{5.95 \mathrm{~km} / \mathrm{s}}=14.5^{\circ}$

Second critical angle for steel by water immersion test

$\operatorname{Arcsin}\left[\frac{V_{\mathrm{LH}_{2} \mathrm{O}}}{V_{\text {Ssteel }}}\right]=\arcsin \frac{1.49 \mathrm{~km} / \mathrm{s}}{3.2 \mathrm{~km} / \mathrm{s}}=27.8^{\circ}$

First critical angle for steel using plexigass wedge

Arcsine $\left[\frac{V_{\text {Lwedge }}}{V_{\text {Lsteel }}}\right]=\arcsin \frac{2.67 \mathrm{~km} / \mathrm{s}}{5.95 \mathrm{~km} / \mathrm{s}}=26.7^{\circ}$

Second critical angle for steel using plexigass wedge 


$$
\operatorname{Arcsin}\left[\frac{V_{\text {Lwedge }}}{V_{\text {Ssteel }}}\right]=\arcsin \frac{2.67 \mathrm{~km} / \mathrm{s}}{3.2 \mathrm{~km} / \mathrm{s}}=56.6^{\circ}
$$

\section{Summary}

Surface (Rayleigh) wave are induced at solid-gas or solid-liquid interfaces, when the probe is at the second critical angle setting; The surface wave profile is mainly transverse and so the surface wave is approximately a shear wave at its critical angle. The variation of sound velocities with frequency is very small (non dispersive) over the frequency range used in NDT.

\section{Acknowledgments}

This work was supported by the Project of Education Department of Jilin Province (Grant No.2014388).

\section{References}

[1] B. X. Zhang, Ph.D. Dissertation (Jilin University) (1994).

[2] B. X. Zhang, and K. X. Wang, J. Acoust. Soc. Am. 992674 (1996).

[3] B. X. Zhang, and K. X. Wang, Chinese J. Geophys. 43749 (2000).

[4] L. Zhang, X. F. Liu, W. Yuan and X. T. Wang, J. Korean. Phys. Soc. 641808 (2014).

[5] B. X. Zhang, J. Acoust. Soc. Am. 96 (4), 2546 (1994). 